\title{
DECOMPOSABLE AND SPECTRAL OPERATORS ON A HILBERT SPACE
}

\author{
BHUSHAN L. WADHWA
}

\begin{abstract}
A necessary and sufficient condition for a decomposable operator, on a Hilbert space, to be equal to a normal plus a commuting quasi-nilpotent operator is found.
\end{abstract}

The notion of a decomposable operator on a Banach space was introduced by Foias [6]. It can be viewed as a nontrivial generalization of the notion of a spectral operator, which is due to Dunford ([2], [3], [4]). The question arises: Under what conditions is a decomposable operator a spectral operator? We do not know the complete answer to this question, but in this note we find a condition which is necessary and sufficient for a decomposable operator on a Hilbert space to be equal to a normal operator plus a commuting quasi-nilpotent operator.

Let $T$ be a bounded linear operator on a Hilbert space $H$. A subspace $M$ of $H$ is called a spectral maximal space of $T$ if (i) $M$ is invariant under $T$ and (ii) if $N$ is any subspace of $H$ invariant under $T$ such that $\sigma(T \mid N) \subset$ $\sigma(T \mid M)$ then $N \subset M$, where $T \mid N$ denotes the restriction of $T$ to the subspace $N$ and $\sigma(T)$ denotes the spectrum of $T$. $T$ is called a decomposable operator if, for every finite open covering $G_{i}, i=1,2, \cdots, n$, of $\sigma(T)$, there exists a family $M_{i}, i=1, \cdots, n$, of spectral maximal spaces of $T$ such that (i) $H=M_{1}+M_{2}+\cdots+M_{n}$, and (ii) $\sigma\left(T \mid M_{i}\right) \subset G_{i}$ for $i=1,2, \cdots, n$. These definitions are due to Foias [6]. Let $x \in H$; then $(T-z I)^{-1} x=R(z, x)$ is an analytic vector-valued function for $z \in$ complement of $\sigma(T)=\rho(T)$. A vector-valued function $f(z)$ is an analytic extension of $R(z, x)$ if it is defined and is analytic on an open set $D(f)$ containing $\rho(T)$ and if $(T-z I) f(z)=x$ for all $z \in D(f) . R(z, x)$ possesses the single-valued extension property if any two extensions of $R(z, x)$ coincide on the common domain. If $R(z, x)$ has the single-valued extension property then we define a maximal singlevalued extension of $R(z, x)$ by taking the union of all extensions of $R(z, x)$ and we designate this by $R_{e}(z, x)$. The operator $T$ is said to have the singlevalued extension property if $R(z, x)$ has the single-valued extension

Presented to the Society, January 17, 1972; received by the editors October 20, 1972 and, in revised form, December 11, 1972.

AMS (MOS) subject classifications (1970). Primary 47B20, 47B40, 47B99.

Key words and phrases. Decomposable operator, spectral operator, spectral maximal space, hyponormal operator.

(c) American Mathematical Society 1973 
property for all $x \in H$. We now define

$$
\begin{aligned}
& \rho(T, x)=\left\{z: R_{e}(z, x) \text { is analytic at } z\right\}, \\
& \sigma(T, x)=[\rho(T, x)]^{\prime}, \text { the complement of } \rho(T, x) .
\end{aligned}
$$

The definitions of $R_{e}(z, x), \rho(T, x), \sigma(T, x)$ are due to Dunford ([2], [3], [4]); the reader will also find a discussion of the properties of $\sigma(T, x)$ there.

The following result is due to Foias [6], and for more results about decomposable operators the reader is referred to Colojoara and Foias [1].

THEOREM F (FoIAS). If $T$ is a decomposable operator on $H$, then:

(i) $T$ has the single-valued extension property.

(ii) For any $\delta \subset \sigma(T), \delta$ closed, $M_{\delta}=\{x \in H: \sigma(T, x) \subset \delta\}$ is a spectral maximal space of $T$, and conversely if $M$ is any spectral maximal space of $T$, then $M=M_{\delta}$ where $\delta=\sigma(T \mid M)$.

A decomposable operator $T$ on a Hilbert space $H$ is said to possess property (I) if, for every closed set $\delta, \sigma\left(T, P_{\delta} x\right) \subset \sigma(T, x)$ for all $x \in H$, where $P_{\delta}$ denotes the projection of $H$ onto $M_{\delta}$.

Proposition. Let $T$ be a decomposable operator with property (I), then $\sigma(T, x) \cap \sigma(T, y)=\varnothing$ implies that $(x, y)=0$.

Proof. Let $\sigma(T, x)=\delta_{1}$ and $\sigma(T, y)=\delta_{2}$. Since $T$ has the property (I), $\sigma\left(T, P_{\delta_{2}} x\right) \subset \sigma(T, x)=\delta_{1}$. But $P_{\delta_{2}} x \in M_{\delta_{2}}$ and hence $\sigma\left(T, P_{\delta_{2}} x\right) \subset \delta_{1} \cap \delta_{2}=\varnothing$. Thus $P_{\delta_{2}} x=0$ and hence $(x, y)=0$.

The property $\sigma(T, x) \cap \sigma(T, y)=\varnothing$ implies $(x, y)=0$ is due to Stampfli [9] and is the orthogonality version of Dunford's boundedness condition (B) [4]. If $T=N+Q$ where $N$ is normal and $Q$ is quasi-nilpotent such that $Q N=N Q$, then $T$ has property (I) (since in this case $P_{\delta}$ will commute with $T)$.

In order to prove our main result we need the following Lemma.

Lemma. Let $T$ be a decomposable operator with property (I). Then for every closed set $\delta, M_{\delta}^{\perp}=\bar{M}_{\delta^{\prime}}$, where $\delta^{\prime}$ is the complement of $\delta$.

Proof. First we shall show that, for any open set $G$ containing $\delta$, $M_{\bar{G}} \subset M_{\delta^{\prime}}$. Since $G$ and $\delta^{\prime}$ cover $\sigma(T)$ and $T$ is decomposable, there exist spectral maximal spaces $Z_{1}$ and $Z_{2}$ of $T$ such that $H=Z_{1}+Z_{2}$ and $\sigma\left(T \mid Z_{1}\right) \subset$ $G \cap \sigma(T)$ and $\sigma\left(T \mid Z_{2}\right) \subset \delta^{\prime} \cap \sigma(T)$. Let $x \in M_{\bar{G}}^{\frac{1}{G}}$ and let $x=z_{1}+z_{2}$ where $z_{i} \in Z_{i}$. Since $Z_{1} \subset M_{\bar{G}}, 0=P_{G} x=z_{1}+P_{\bar{G}^{z}} z_{2}$. Thus $\sigma\left(T, z_{1}\right)=\sigma\left(T, P_{G^{\prime}} z_{2}\right) \subset$ $\bar{G} \cap \sigma\left(T, z_{2}\right)$ (because $T$ has the property $\left.(\mathrm{I})\right) \subset \bar{G} \cap \delta^{\prime}$. Hence $\sigma(T, x) \subset$ $\sigma\left(T, z_{1}\right) \cup \sigma\left(T, z_{2}\right) \subset \delta^{\prime}$. Thus $M_{\delta^{\prime}}^{\frac{1}{\delta^{\prime}} \subset M_{G}}$ for any open set $G \supset \delta$. Now by Foias [7], $M_{\delta}=\cap M_{\bar{G}}$ where the intersection is taken over all open sets $G$ containing $\delta$. Hence $M_{\delta^{\prime}}^{\perp} \subset M_{\delta}$ and by the proposition $\bar{M}_{\delta^{\prime}} \subset M_{\delta}^{\perp}$. 
THEOREM. Let $T$ be a decomposable operator with property (I). Then $T=N+Q$ where $N$ is normal, $Q$ is quasi-nilpotent and $N Q=Q N$.

Proof. For this proof we shall use the notation and the terminology of Dunford and Schwartz [5, pp. 2136-2149]. From the Theorem F and the Proposition it follows that $T$ satisfies Dunford's conditions (A), (B) and (C). In order to show that $T$ satisfies condition (D), from the Lemma it follows that for any closed set $\delta, H=M_{\delta} \oplus \bar{M}_{\delta^{\prime}}$ and hence $\delta \in S_{1}(T)$. Since $\delta^{\prime}$ is open, there is an increasing sequence of closed sets $\beta_{n}$ such that $\delta^{\prime}=$ $\bigcup \beta_{n}$. For any $x \in M_{\delta^{\prime}}$, since $\sigma(T, x)$ is compact, $\sigma(T, x) \subset \beta_{n}$ for large values of $n$. Hence each closed set $\delta$ is in $S(T)$. Now by Theorem XVI.4.5 [5], $T$ is a spectral operator and, by Stampfli [9, Lemma 7], $T$ has a resolution of identity consisting of selfadjoint projections, i.e., $T=N+Q$ where $N$ is normal, $Q$ is quasi-nilpotent and $N Q=Q N$.

REMARKS. The author, in [10], proved by a different method, that a hyponormal decomposable operator with property $(\mathrm{I})$ is normal. This result now easily follows from the Theorem. Putnam [8, p. 476] has shown the existence of hyponormal nonnormal operators with totally disconnected spectrum. It is a fact that operators with totally disconnected spectrum are decomposable. Thus a decomposable hyponormal operator is not necessarily normal.

\section{REFERENCES}

1. I. Colojoara and C. Foias, Theory of generalized spectral operators, Gordon and Breach, New York, 1968.

2. N. Dunford, Spectral theory. II. Resolution of identity, Pacific J. Math. 2 (1952), 559-614. MR 14, 479.

3. - Spectral operators, Pacific J. Math. 4 (1954), 321-354. MR 16, 142.

4. - A survey of the theory of spectral operators, Bull. Amer. Math. Soc. 64 (1958), 217-274. MR 21 \#3616.

5. N. Dunford and J. T. Schwartz, Linear operators. III. Spectral operators, WileyInterscience, New York, 1971.

6. C. Foias, Spectral maximal spaces and decomposable operators in Banach spaces, Arch. Math. 14 (1963), 341-349. MR 27 \#2865.

7. - Spectral capacities and decomposable operators, Rev. Roumaine Math. Pures Appl. 13 (1968), 1539-1545. MR 40 \#3334.

8. C. R. Putnam, The spectra of subnormal operators, Proc. Amer. Math. Soc. 28 (1971), 473-477. MR 43 \#972.

9. J. G. Stampfli, Analytic extensions and spectral localization, J. Math. Mech. 16 (1966), 287-296. MR 33 \#4687.

10. B. L. Wadhwa, Spectral, M-hyponormal and decomposable operators, Dissertation, Indiana University, 1971.

Department of Mathematics, Cleveland State University, Cleveland, Ohio 44115 